\title{
State of the art in the management of oral squamous cell carcinoma
}

\author{
Raúl González-García
}

Department of Oral and Maxillofacial-Head and Neck Surgery, University Hospital Infanta Cristina, 06080 Badajoz, Spain. Correspondence Author: Prof. Raúl González-García, Department of Oral and Maxillofacial-Head and Neck Surgery, University Hospital Infanta Cristina, 06080 Badajoz, Spain. E-mail: raulmaxilo@gmail.com

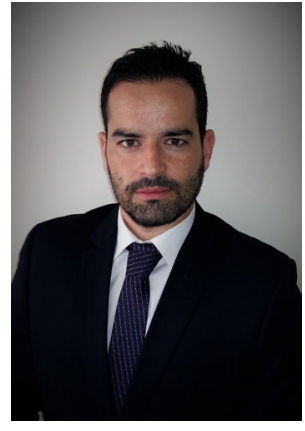

Dr. Raúl González-García, M.D., Ph.D, FEBOMFS, was graduated in Medicine (Medical Degree, M.D.) by the Universidad Autónoma de Madrid School of Medicine, Madrid, Spain, in 2001, and obtained his trainee in Oral and Maxillofacial Surgery in the University Hospital La Princesa, Madrid, Spain, in 2007. He obtained his Ph.D. Degree in Medicine and Surgery at the Universidad Autónoma de Madrid School of Medicine, in 2011. Since 2007 he is Consultant Surgeon at the Department of Oral and Maxillofacial Surgery, University Hospital Infanta Cristina, Badajoz, Spain, and he is also a University Honorary Collaborator at the Universidad de Extremadura School of Medicine. He is actually Editor-in-Chief of Plastic and Aesthetic Research, Assistant Director of Revista Española de Cirugía Oral y Maxilofacial, Permanent Member of the Reviewer Commitee of International Journal of Oral and Maxillofacial Surgery, Contributing Editor of Current Research in Dentistry, and Member of the Editorial Board/reviewer of more than 35 international journals of the speciality. He is author of more than 130 publications, 96 of them in international Medline/PubMed-indexed journals, 15 chapters in books of the speciality, and he is author of the text book "Reconstrucción Maxilomandibular Compleja: Microcirugía, Distracción Ósea e Implantes dentales".

When considering the last report by the World Health Organization in 2014, oral squamous cell carcinoma (OSCC) represents the seventh cause of cancer worldwide and causes 529,000 new cases per year. In addition to its considerable frequency, death by this entity may overcome in approximately $50 \%$ of the patients, even after aggressive multi-modality treatment. Although surgery followed or not by radiotherapy is the main treatment option, many factors may influence overall outcome in terms of survival, including patient-related, tumor-related and treatment-related factors.

A myriad of articles has been published in the literature concerning "oral cancer" and "squamous cell carcinoma of the oral cavity and oropharynx". Although several highquality papers are advised, the heterogeneity of the studies in terms of methodology, treatment protocols, and inclusion/ exclusion criteria makes direct comparison among series and institutions challenging. However, some main guidelines may guide the clinician into the best treatment modality for each patient, while an effort in terms of classifying patients who may benefit from one or other treatment option has been made in the last decade.

\begin{tabular}{|l|l|}
\hline \multicolumn{2}{|c|}{ Access this article online } \\
\hline Quick Response Code: & Website: \\
\hline & http://www.parjournal.net \\
\cline { 2 - 3 } & \\
\hline
\end{tabular}

The present editorial is an introductory paper to a special issue composed of eleven manuscripts focusing on particular aspects about the state of the art in OSCC under the light of current knowledge. While strict criteria for systematic reviewing concerning Cochrane database recommendations have not been followed due to the heterogeneity of the revised series, an effort has been made to selectively approach particular aspects of OSCC management, while selecting the most prominent series within the last 15 years. Some of the incoming works have made a special effort in trying to include and systematically analyze more relevant works according to a predetermined search, inclusion and exclusion criteria, with a particular PICO question and a flow chart, while others have made a general review according to subjective criteria based on authors' own experience in selecting more relevant works. While the more systematically approach is more relevant in the evidence-based scale, reviews by selected experts still have a relevant role in the field of surgery.

The approached items were: (1) the role of human

This is an open access article distributed under the terms of the Creative Commons Attribution-NonCommercial-ShareAlike 3.0 License, which allows others to remix, tweak and build upon the work non-commercially, as long as the author is credited and the new creations are licensed under the identical terms.

For reprints contact: service@oaepublish.com

How to cite this article: González-García R. State of the art in the management of oral squamous cell carcinoma. Plast Aesthet Res 2016;3:129-31.

Received: 20-04-2016; Accepted: 04-05-2016 

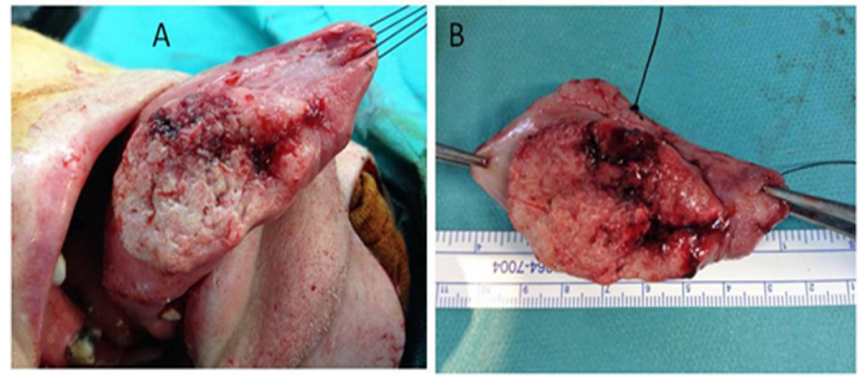

Figure 1: Oral squamous cell carcinoma (OSCC) of the tongue. (A) Intraora view of the tumor; (B) immediate intraoperative tissue shrinkage following resection of OSCC by a partial glossectomy
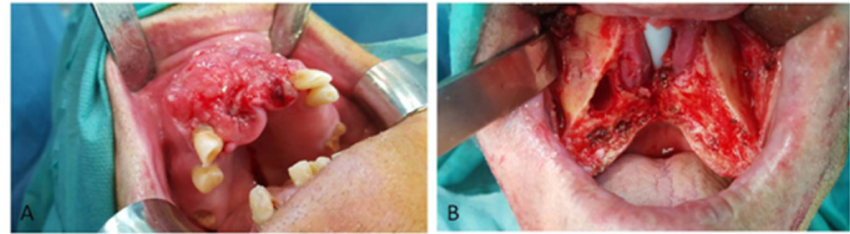

Figure 3: Oral squamous cell carcinoma of the upper maxilla. (A) Intraoral view of the tumor; (B) intraoperative view of the remnant maxilla following anterior partial maxillectomy

papillomavirus (HPV) in OSCC; (2) the role of sentinel node biopsy in the treatment of OSCC; (3) the influence of surgical resection margins in OSCC; $(4)$ the role of radiotherapy in OSCC; (5) the role of elective neck dissection in early-staged OSCC; (6) the role of elective neck dissection in OSCC of the upper maxilla; (7) the role of contralateral neck dissection in OSCC; (8) the role of salvage surgery in recurrent OSCC; (9) the reliability of the combined-triangular full-thickness skin graft for covering the radial forearm free flap donorsite; (10) the prognosis and quality of life in head and neck cancer; and (11) last but not least a special article about the current role of the facial allograft transplantation. This last paper is performed by one of the first and more experienced surgical teams in performing facial allograft transplantation worldwide.

Electronic literature search was conducted mostly in Medline, but also Embase and Cochrane databases were used in some of the articles in the present issue. The abstracts of yielded results were reviewed and the full text of those with apparent relevance was obtained. The references of identified articles were crosschecked for unidentified articles, and only those in English language were selected. In most of the articles in the present issue, exclusion criteria concerning the type of articles to be included were established, thus excluding case reports and technical notes, letters to Editors, expert opinions, animal or in vitro studies, review articles, and repetitive data from series of the same authors or institutions. Qualitative systematic reviews or non-systematic reviews were performed, while two articles, one concerning elective neck dissection in OSCC of the upper maxilla, and the other dealing with salvage surgery for OSCC, analyzed data from features in the selected articles to infer new results as meta-analysis. Two additional articles are dealing with special reconstructive surgical procedures that were developed and put in practice by the authors' surgical teams, being the facial allograft transplantation and the covering of the radial forearm free flap donor-site by the

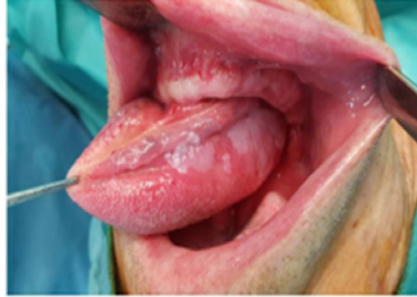

A

Figure 2: Selective supraomohioid neck dissection involving cervical levels I to III in a T1 oral squamous cell carcinoma of the tongue. (A) Intraoral view of the tumor; (B) intraoperative view of the dissected neck

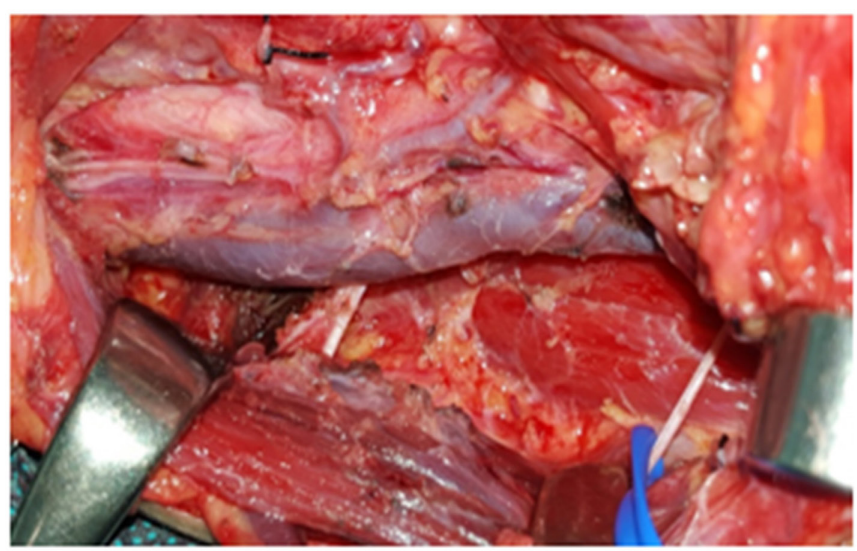

Figure 4: Contralateral modified type III radical neck dissection in an oral squamous cell carcinoma of the mandible involving the right parasymphiseal region. Detail of levels II to $\mathrm{V}$ left neck dissection

elsewhere named "Iberic graft", a new technique consisting on the use of full-thickness skin grafts obtained from the volar side of the forearm skin.

Some strong conclusions can be obtained from the reading of the reviewing works included in this special issue, which may move the reader closer to the current knowledge about OSCC, or at least may provide a baseline from which the reader can deep into a more specific and advanced knowledge. These conclusions are: (1) HPV is increasingly being associated to OSCC, usually diagnosed at a younger age, mainly in the oropharynx, while $\mathrm{HPV}^{+}$OSCC patients have an increased survival, better treatment response and lower recurrence rates; (2) the sentinel node biopsy is a reliable staging method in early-stage OSCC as an alternative to elective neck dissection for staging N0-neck, with a reasonable false-negative rate while decreasing morbidity associated to selective neck dissection if performed by an experienced multidisciplinary team; (3) tissue shrinkage on surgical margins of resection in OSCC is a tangible phenomenon, being the highest percentage of retraction occurring at the time of resection, which leads the surgeon into a major therapeutic role, as involved or closed margins in the histologic report may determine the administration of complementary treatments such as post-operative radiotherapy and/or chemotherapy [Figure 1]; (4) both external radiotherapy and brachytherapy play a determinant role in the treatment of OSCC, alone in early stages or combined with surgery and/or chemotherapy in advanced ones; (5) if routine strict follow-up using ultra-sonography fine needle aspiration citology (USgFNAC) by a well-trained ultrasonographer cannot be assured in clinically N0-neck, 


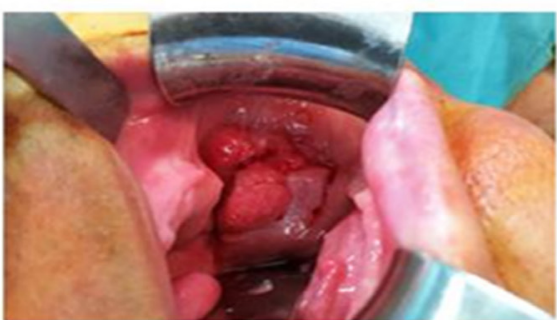

A

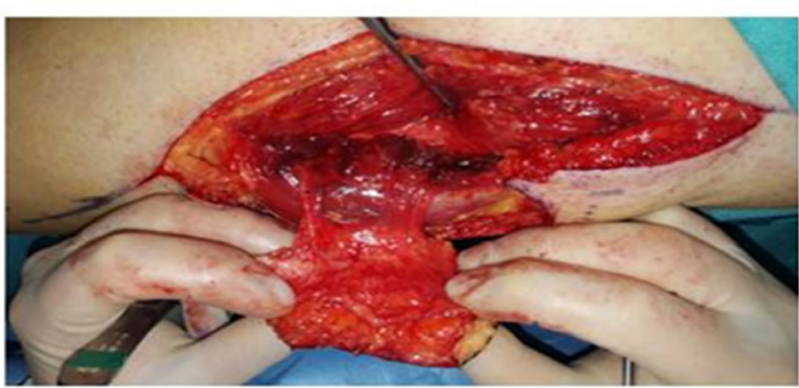

C

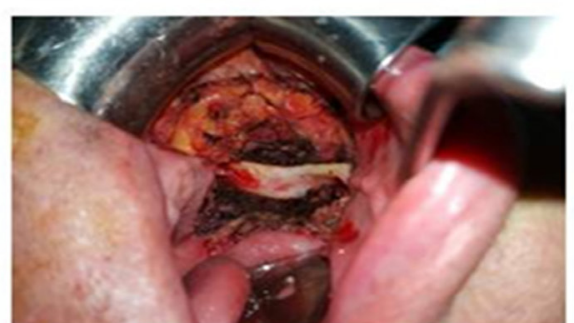

B

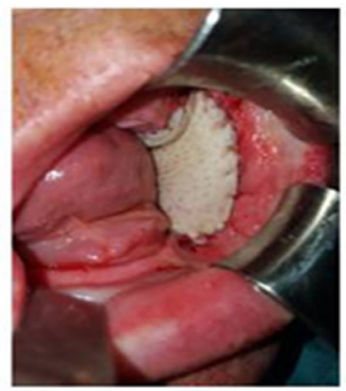

D

Figure 5: Recurrent oral squamous cell carcinoma of the buccal mucosa. (A) Intraoral view of the tumor; (B) intraoperative view of the resection; (C) reconstruction with an antero-lateral thigh flap; (D) intraoperative view of the reconstructed defect

elective neck dissection is advised by a supraomohyoid neck dissection as an effective method to control cervical metastases [Figure 2]; (6) the rate of metastases in patients with OSCC of the upper maxilla is high and comparable with metastases from other sites of the oral cavity, thus elective neck dissection should be routinely performed in T3/T4 tumors of the upper maxilla [Figure 3]; (7) with a variable reported contralateral lymph neck node metastases rate in OSCC ranging from $0.9 \%$ to $36 \%$, surgeons should take into account the detailed and individual study of risks and potential benefits of elective neck dissection for contralateral N0 neck [Figure 4]; (8) from a meta-analysis of 13 eligible series comprising 1,692 recurrent OSCC patients, recurrence appeared in up to $26 \%$ of primarily treated patients. With a mean 5 -year overall survival rate of $40.2 \%$, salvage surgery is the first treatment option for recurrent OSCC patients [Figure 5]; (9) the "Iberic graft", consisting on a combined full-thickness skin graft obtained from the volar forearm skin, is a reliable method for closing most of radial forearm free flap donor-site defects, as it provides excellent color match and pliability, while obviates the need for a second surgical site; (10) although prognosis of OSCC has improved, further studies are necessary to understand the behaviour in every case and determine how the impact on the quality of life can be a useful tool to individualize therapies; and (11) although clinical experience has demonstrated the facial transplantation viability, it is still considered an experimental procedure in which we have much to learn to define its true role in the current reconstructive surgery and resolve major technical, medical and ethical problems involved.

In summary, these and several other conclusions can be highlighted from the reviews of this special issue. OSCC represents a challenge for the clinician as mortality and morbidity still remain high in advance-stage patients, despite recent advances in therapies and reconstructive options. Surgery still plays the major role in controlling the disease and provides the highest overall and specific-disease survival rates, although advanced-stage disease may require the administration of postoperative radiotherapy with or without chemotherapy.

This is the reason why a multidisciplinary approach with surgeons, radiotherapy oncologists, medical oncologists and others is mandatory to obtain the highest standards in terms of survival and quality of life, with the Head and Neck Surgeon leading this team. The acquisition of new knowledge in relation to OSCC may add new weapons to the armamentarium of the clinician. This and other compendiums of current knowledge about the disease may establish the baseline for further goals and achievements in the battle against oral cancer.

\section{Financial support and sponsorship}

Nil.

\section{Conflicts of interest}

There are no conflicts of interest. 